\title{
UNIFORM DISTRIBUTION OF SECOND-ORDER LINEAR RECURRING SEQUENCES
}

\author{
G. TURNWALD
}

\begin{abstract}
A complete classification is obtained for all second-order linear recurring sequences uniformly distributed modulo an ideal of a Dedekind domain.
\end{abstract}

1. Introduction. A sequence of rational integers is said to be uniformly distributed modulo $m$ (u.d. $\bmod m$ ) if every residue class appears with the same asymptotic frequency. Uniform distribution of second-order linear recurring sequences was first investigated in special cases $[4,5,9]$; then several authors obtained (partial) results concerning u.d. modulo prime powers $[2,6,8,16]$. Finally, R. T. Bumby provided a complete solution [1]; cf. [7, Chapter III]. The corresponding problem for order three was solved by Knight and Webb under the additional hypothesis that $m$ is relatively prime to 2,3,5 [3]. Bumby remarks that his methods could possibly lead to a solution of the corresponding problem with algebraic integers. This is indeed the case (and was carried out by the author; unpublished), but the approach presented here leads to considerably more general results. Specializing to rational integers, our method yields an elementary proof of Bumby's result, totally avoiding algebraic number theory. It is also possible to obtain a characterization of u.d. third-order linear recurring sequences in a similar way; the result, however, becomes very complicated [15]. The special case of rational integers (formulated in [13]) completes the investigations of Knight and Webb. The method presented here also leads to partial results concerning u.d. modulo prime powers for linear recurring sequences of arbitrary order [13, 15]. Uniform distribution modulo a prime ideal (of finite norm) in a Dedekind domain amounts to u.d. in a finite field, which was investigated by Niederreiter and Shiue for linear recurring sequences up to order four [10, 11].

This paper is distilled from my thesis [14], written under the supervision of Dozent R. F. Tichy. The case of order two that is treated here was excluded in [15], where the larger part of the thesis is published. (The results of $\$ \S 2$ and 3 essentially appear in [15] too, but mostly without (complete) proof.)

2. Linear recurring sequences. Let $R$ be a commutative ring with unit element; let $\left(u_{n}\right)$ be a sequence of elements of $R$. A polynomial $\sum a_{k} x^{k}$ with coefficients in $R$ is

Received by the editors March 5, 1985.

1980 Mathematics Subject Classification. Primary 10A35, 12A05.

Key words and phrases. Linear recurring sequences, uniform distribution in residue classes, algebraic integers, $p$-adic integers.

- 1986 American Mathematical Society $0002-9939 / 86 \$ 1.00+\$ .25$ per page 
called a characteristic polynomial of $\left(u_{n}\right)$ if $\sum a_{k} u_{n+k}=0$ for all $n \geqslant 0$. If $a(x)=$ $\sum a_{k} x^{k}$ is a characteristic polynomial and $b(x)=\sum b_{k} x^{k}$ is an arbitrary polynomial, then $a(x) b(x)$ again is a characteristic polynomial of $\left(u_{n}\right)$, since

$$
\sum_{i, j} a_{i} b_{j} u_{n+i+j}=\sum_{j} b_{j}\left(\sum_{i} a_{i} u_{n+i+j}\right)=0 .
$$

If $\left(u_{n}\right)$ admits a monic characteristic polynomial $c(x)$, we call $\left(u_{n}\right)$ a linear recurring sequence with characteristic polynomial $c(x)$. We do not require that $c(x)$ has minimal degree. Even a restriction to monic characteristic polynomials of minimal degree would not guarantee uniqueness: In a ring of characteristic 4 the sequence $(2,2,0,2,2,0, \ldots)$ admits the different monic characteristic polynomials $x^{2}-x-1$ and $x^{2}+x+1$ of degree two, but none of degree one. In a unique factorization domain, however, uniqueness can be proven [14, Proposition 1.1.4]. By an $r$ th-order linear recurring sequence we mean a sequence that admits a monic characteristic polynomial of degree $r$; again, no minimality condition is assumed. Such a sequence $\left(u_{n}\right)$ is uniquely determined by a characteristic polynomial of degree $r$ and the initial terms $u_{0}, \ldots, u_{r-1}$.

LEMMA 1. Let $I$ be an ideal of $R$ and let $\left(u_{n}\right)$ be a linear recurring sequence with characteristic polynomial $c(x)$. If $\sum a_{k} x^{k} \equiv 0(c(x), I)$ then $\sum a_{k} u_{n+k} \equiv 0(I)$ for all $n \geqslant 0$.

Proof. By assumption there exists a polynomial $\sum b_{k} x^{k}$ with coefficients in $I$ such that $\sum\left(a_{k}-b_{k}\right) x^{k}$ is a multiple of $c(x)$. Hence, $\Sigma\left(a_{k}-b_{k}\right) u_{n+k}=0$ and $\sum a_{k} u_{n+k}$ $\equiv \sum\left(a_{k}-b_{k}\right) u_{n+k} \equiv 0(I)$.

For the following calculations it is useful to observe that the congruences $f(x) \equiv 0(c(x), I)$ and $g(x) \equiv 0(c(x), J)$ imply $f(x) g(x) \equiv 0(c(x), I J)$.

LemMA 2. Assume that $x^{n}\left(x^{l}-1\right) \equiv 0(c(x), I)$. Then

$$
x^{2 n}\left(x^{k l}-1\right) \equiv x^{2 n} k\left(x^{l}-1\right)\left(c(x), I^{2}\right)
$$

and

$$
x^{3 n}\left(x^{k l}-1\right) \equiv x^{3 n} k\left(x^{l}-1\right)+x^{3 n}\left(\begin{array}{l}
k \\
2
\end{array}\right)\left(x^{l}-1\right)^{2}\left(c(x), I^{3}\right)
$$

for $k>0$.

Proof. From

$$
x^{k l}-1=\left(1+\left(x^{l}-1\right)\right)^{k}-1=\sum_{j=1}^{k}\left(\begin{array}{l}
k \\
j
\end{array}\right)\left(x^{l}-1\right)^{j}
$$

and $x^{2 n}\left(x^{l}-1\right)^{2} \equiv 0\left(c(x), I^{2}\right)$ we deduce

$$
x^{2 n}\left(x^{k l}-1\right) \equiv x^{2 n}\left(\begin{array}{c}
k \\
1
\end{array}\right)\left(x^{l}-1\right)\left(c(x), I^{2}\right) .
$$

Analogously, the second assertion follows from $x^{3 n}\left(x^{l}-1\right)^{3} \equiv 0\left(c(x), I^{3}\right)$. 
THEOREM 1. Let $I$ be an ideal of $R$ and assume that $a \equiv 0(I)$ for a positive integer a. If $x^{n_{0}}\left(x^{l}-1\right) \equiv 0\left(c(x), I^{h_{0}}\right)\left(n_{0} \geqslant 0 ; l, h_{0}>0\right)$ then for every linear recurring sequence $\left(u_{n}\right)$ with characteristic polynomial $c(x)$ we have

(a) $u_{n+a^{\prime \prime l}} \equiv u_{n}\left(I^{h_{0}+h}\right)$ for $h \geqslant 0, n \geqslant 2^{h} n_{0}$,

(b) $u_{n+k l} \equiv u_{n}+k\left(u_{n+l}-u_{n}\right)\left(I^{2 h_{0}}\right)$ for $n \geqslant 2 n_{0}$,

(c) $u_{n+k a^{h l}} \equiv u_{n}+k a^{h-1}\left(u_{n+a l}-u_{n}\right)\left(I^{2 h_{0}+h}\right)$ for $h>0, n \geqslant 3 \cdot 2^{h-1} n_{0}$,

(d) $u_{n+k a^{h l}} \equiv u_{n}+k a^{h}\left(u_{n+l}-u_{n}\right)\left(I^{2 h_{0}+h}\right)$ for $h>0, n \geqslant 3 \cdot 2^{h-1} n_{0}$, a odd.

Proof. By Lemma 1 it suffices to show that

(a') $x^{2^{h} n_{0}}\left(x^{a^{h l} l}-1\right) \equiv 0\left(c(x), I^{h_{0}+h}\right)$,

(b') $x^{2 n_{0}}\left(x^{k l}-1\right) \equiv x^{2 n_{0}} k\left(x^{l}-1\right)\left(c(x), I^{2 h_{0}}\right)$,

(c') $x^{3 \cdot 2^{h-1} n_{0}}\left(x^{k a^{h l}}-1\right) \equiv x^{3 \cdot 2^{h-1} n_{0}} k a^{h-1}\left(x^{a l}-1\right)\left(c(x), I^{2 h_{0}+h}\right)$,

$\left(\mathrm{d}^{\prime}\right) x^{3 \cdot 2^{h-1} n_{0}}\left(x^{k a^{h} l}-1\right) \equiv x^{3 \cdot 2^{h-1} n_{0}} k a^{h}\left(x^{\prime}-1\right)\left(c(x), I^{2 h_{0}+h}\right)$.

To simplify the notation we write $n$ instead of $n_{0}$ in the sequel.

From $x^{2^{h} n}\left(x^{a^{h} l}-1\right) \equiv 0\left(c(x), I^{h_{0}+h}\right)$ we conclude that

$$
x^{2^{h+1} n}\left(x^{a^{h+1} l}-1\right) \equiv x^{2^{h+1} n} a\left(x^{a^{h} l}-1\right)\left(c(x), I^{2 h_{0}+2 h}\right)
$$

by Lemma 2 . Since $x^{2^{h} n} a\left(x^{a^{h} l}-1\right) \equiv 0\left(c(x), I^{h_{0}+h+1}\right)$ and $h_{0}+h+1 \leqslant 2 h_{0}+$ $2 h$, this proves $\left(\mathrm{a}^{\prime}\right)$ by induction, the case $h=0$ being trivial. Lemma 2 , again, proves $\left(b^{\prime}\right)$.

Since $2 h_{0}+1 \leqslant 3 h_{0}$, Lemma 2 shows that

$$
x^{3 n}\left(x^{k a l}-1\right) \equiv x^{3 n} k\left(x^{a l}-1\right)+x^{3 n}\left(\begin{array}{l}
k \\
2
\end{array}\right)\left(x^{a l}-1\right)^{2}\left(c(x), I^{2 h_{0}+1}\right) .
$$

From $x^{n}\left(x^{a l}-1\right) \equiv 0\left(c(x), I^{h_{0}}\right)$ and $x^{2 n}\left(x^{a l}-1\right) \equiv 0\left(c(x), I^{h_{0}+1}\right)($ case $h=1$ of $\left.\left(a^{\prime}\right)\right)$ we conclude

$$
x^{3 n}\left(x^{a l}-1\right)^{2} \equiv 0\left(c(x), I^{2 h_{0}+1}\right) .
$$

This gives case $h=1$ of $\left(\mathrm{c}^{\prime}\right)$. By $\left(\mathrm{a}^{\prime}\right)$ we have $x^{2^{h} n}\left(x^{k a^{h} l}-1\right) \equiv 0\left(c(x), I^{h_{0}+h}\right)$ so that, by Lemma 2 ,

$x^{3 \cdot 2^{h} n}\left(x^{a k a^{h} l}-1\right) \equiv x^{3 \cdot 2^{h} n} a\left(x^{k a^{h} l}-1\right)+x^{3 \cdot 2^{h} n}\left(\begin{array}{l}a \\ 2\end{array}\right)\left(x^{k a^{h} l}-1\right)^{2}\left(c(x), I^{3\left(h_{0}+h\right)}\right)$.

Since $x^{2 \cdot 2^{h} n}\left(x^{k a^{h} l}-1\right)^{2} \equiv 0\left(c(x), I^{2\left(h_{0}+h\right)}\right)$ and $2 h_{0}+h+1 \leqslant 2\left(h_{0}+h\right)$, the second term vanishes $\bmod I^{2 h_{0}+h+1}$, and we obtain

$$
x^{3 \cdot 2^{h} n}\left(x^{k a^{h+1} l}-1\right) \equiv x^{3 \cdot 2^{h} n} a\left(x^{k a^{h} l}-1\right)\left(c(x), I^{2 h_{0}+h+1}\right) .
$$

The proof of $\left(\mathrm{c}^{\prime}\right)$ now follows by induction since $a \equiv 0(I)$.

If $a$ is odd, then $\left(\begin{array}{l}a \\ 2\end{array}\right)=a(a-1) / 2 \equiv 0(I)$. Hence

$$
x^{3 n}\left(\begin{array}{l}
a \\
2
\end{array}\right)\left(x^{l}-1\right)^{2} \equiv 0\left(I^{2 h_{0}+1}\right)
$$

and Lemma 2 gives

$$
x^{3 n}\left(x^{a l}-1\right) \equiv x^{3 n} a\left(x^{l}-1\right)+x^{3 n}\left(\begin{array}{l}
a \\
2
\end{array}\right)\left(x^{l}-1\right)^{2} \equiv x^{3 n} a\left(x^{l}-1\right)\left(c(x), I^{2 h_{0}+1}\right) .
$$


Since $a^{h-1} \equiv 0\left(I^{h-1}\right)$, this implies

$$
x^{3 \cdot 2^{h-1} n} a^{h-1}\left(x^{a l}-1\right) \equiv x^{3 \cdot 2^{h-1} n} a^{h}\left(x^{l}-1\right)\left(c(x), I^{2 h_{0}+h}\right) .
$$

Now $\left(\mathrm{d}^{\prime}\right)$ follows from $\left(\mathrm{c}^{\prime}\right)$.

3. Uniform distribution. From now on we assume that $R$ is a Dedekind domain, i.e., an integral domain in which every nonzero ideal admits a (unique) representation as a product of prime ideals. Equivalently, a Dedekind domain may be defined to be a Noetherian integrally closed domain where every nonzero prime ideal is maximal. The examples we have in mind are $p$-adic integers or the ring of integers in an algebraic number field (of finite degree). We define the norm of an ideal $I$ by $N(I)=|R / I|$. If $I$ and $J$ are ideals with finite norm, then $N(I J)=N(I) N(J)$ (cf. [12, Chapter 8, A]). By the corresponding (rational) prime $p$ of a nonzero prime ideal $P$, we mean the characteristic of the field $R / P$. If $N(P)$ is finite, it is a power of $p$.

A sequence $\left(u_{n}\right)$ of elements of $R$ is called uniformly distributed (u.d.) $\bmod I$ if $I$ is an ideal of finite norm and

$$
\lim _{n \rightarrow \infty} n^{-1}\left|\left\{k \mid 0 \leqslant k<n, u_{k} \equiv x(I)\right\}\right|=1 / N(I)
$$

for every element $x$ of $R$. If $\left(u_{n}\right)$ is u.d. $\bmod I$ and $I$ is contained in $J$, then $\left(u_{n}\right)$ is u.d. $\bmod J$, since every residue class $\bmod J$ consists of $N(I) / N(J)$ residue classes $\bmod I$. If $\left(u_{n}\right)$ is periodic $\bmod I$ and u.d. $\bmod I$, then every period must be divisible by $N(I)$.

Let $\left(u_{n}\right)$ be a linear recurring sequence with characteristic polynomial $c(x)$. We assume that $c(x)$ splits into linear factors modulo $P$ and that all factors incongruent to $x$ occur with multiplicity at most two; $P$ denotes a fixed prime ideal of $R$.

Lemma 3. (a) If $\left(u_{n}\right)$ is $u . d . \bmod P$, then $N(P)=p$,

$$
u_{j+n(p-1)} \equiv u_{j}+n\left(u_{j+p-1}-u_{j}\right)(P), \quad u_{j+p-1}-u_{j} \neq 0(P) \quad \text { for } j \geqslant j_{0},
$$

and $u_{j+p^{h}(p-1)} \equiv u_{j}\left(P^{h}\right)$ for $j \geqslant j_{0}(h)$ and $h \geqslant 1$.

(b) If $N(P)=p$ and $p>2$, then $u_{j+n p^{h}(p-1)} \equiv u_{j}+n p^{h-1}\left(u_{j+p(p-1)}-u_{j}\right)\left(P^{h+1}\right)$ for $j \geqslant j_{0}(h)$ and $h \geqslant 1$.

(c) If $N(P)=p$ and $p \geqslant 5$, then $u_{j+p(p-1)} \equiv u_{j}+p\left(u_{j+p-1}-u_{j}\right)\left(P^{2}\right)$ for $j \geqslant j_{0}$.

Proof. By definition of u.d. $\bmod P, N(P)$ is finite. Let $n_{0}$ be the multiplicity of $x$ in the factorization of $c(x)$ modulo $P$. Then setting $q=N(P)$ we have

$$
x^{n_{0}}\left(x^{q-1}-1\right)^{2} \equiv 0(c(x), P),
$$

since every linear factor incongruent to $x$ is a divisor of $x^{q-1}-1$ and the multiplicity is assumed to be at most 2 . Hence,

$$
x^{n_{0}}\left(x^{p(q-1)}-1\right) \equiv x^{n_{0}}\left(x^{q-1}-1\right)^{p} \equiv 0(c(x), P) .
$$

By Lemma 1 this implies that $\left(u_{n}\right)$ has period $p(q-1)$ modulo $P$. If $\left(u_{n}\right)$ is u.d. $\bmod P$ every period length must be divisible by $q=N(P)$; hence, we conclude $q=p$. From

$$
x^{n_{0}}\left(x^{n(p-1)}-1\right)=x^{n_{0}}\left(\left(1+\left(x^{p-1}-1\right)\right)^{n}-1\right)=x^{n_{0}} \sum_{j=1}^{n}\left(\begin{array}{l}
n \\
j
\end{array}\right)\left(x^{p-1}-1\right)^{j}
$$


and

$$
x^{n_{0}}\left(x^{p-1}-1\right)^{2} \equiv 0(c(x), P)
$$

we deduce

$$
x^{n_{0}}\left(x^{n(p-1)}-1\right) \equiv x^{n_{0}} n\left(x^{p-1}-1\right)(c(x), P) .
$$

Again, by Lemma 1 this implies $u_{j+n(p-1)}-u_{j} \equiv n\left(u_{j+p-1}-u_{j}\right)(P)$ for $j \geqslant j_{0}=$ $n_{0}$. We now apply Theorem 1 (with $I=P, a=p, h_{0}=1, l=p(p-1)$ ) to obtain (after a change of notation)

$$
u_{j+p^{h}(p-1)} \equiv u_{j}\left(P^{h}\right) \text { for } h \geqslant 1, j \geqslant j_{0}(h)
$$

and

$$
u_{j+n p^{h}(p-1)} \equiv u_{j}+n p^{h-1}\left(u_{j+p(p-1)}-u_{j}\right)\left(P^{h+1}\right) \quad \text { for } h \geqslant 1, j \geqslant j_{0}(h), p>2 .
$$

If $u_{j+p-1}-u_{j} \equiv 0(P)$ for some $j \geqslant j_{0}$, from $u_{j+n(p-1)}-u_{j} \equiv n\left(u_{j+p-1}-u_{j}\right)(P)$ we see that the residue $u_{j} \bmod P$ appears at least $p$ times (for $n=0, \ldots, p-1$ ) in a period of length $p(p-1)$; but if $\left(u_{n}\right)$ is u.d. $\bmod P$ every residue must appear $p-1$ times, since the number of residues is $p=N(P)$. This concludes the proof of (a) and (b). To prove (c) we first remark that

$$
\begin{aligned}
x^{2 n_{0}}\left(x^{p(p-1)}-1\right) & =x^{2 n_{0}} \sum_{j=1}^{p}\left(\begin{array}{l}
p \\
j
\end{array}\right)\left(x^{p-1}-1\right)^{j} \\
& \equiv x^{2 n_{0}}\left(p\left(x^{p-1}-1\right)+\left(x^{p-1}-1\right)^{p}\right)\left(c(x), P^{2}\right),
\end{aligned}
$$

since $\left(\begin{array}{c}p \\ j\end{array}\right) \equiv 0(P)$ for $1 \leqslant j \leqslant p-1$ and $x^{n_{0}}\left(x^{p-1}-1\right)^{2} \equiv 0(c(x), P)$. For $p \geqslant 5$ we have $x^{2 n_{0}}\left(x^{p-1}-1\right)^{p} \equiv 0\left(c(x), P^{2}\right)$; hence,

$$
x^{2 n_{0}}\left(x^{p(p-1)}-1\right) \equiv x^{2 n_{0}} p\left(x^{p-1}-1\right)\left(c(x), P^{2}\right),
$$

which implies $u_{j+p(p-1)}-u_{j} \equiv p\left(u_{j+p-1}-u_{j}\right)\left(P^{2}\right)$ for $j \geqslant 2 n_{0}$.

REMARK. If $c(0) \neq 0(P)$, then we may take $n_{0}=0$; hence the conditions given in the lemma hold for $j \geqslant 0$.

LEMMA 4. Assume that $p=2$ and $\left(u_{n}\right)$ is $u . d . \bmod P^{2}$. If $p \equiv 0\left(P^{2}\right)$, then $\left(u_{n}\right)$ is not u.d. $\bmod P^{3}$; if $p \neq \equiv 0\left(P^{2}\right),\left(u_{n}\right)$ is u.d. $\bmod P^{h}$ for all $h \geqslant 1$.

Proof. As in the proof of the preceding lemma, we conclude that $x^{n_{0}}(x-1)^{2} \equiv 0$ $(c(x), P)$, since $\left(u_{n}\right)$ is u.d. $\bmod P$. We define $r(x)=\sum r_{k} x^{k}$ to be the residue of $x^{n_{0}}\left(x^{2}-1\right)$ modulo $c(x)$. From $x^{n_{0}}\left(x^{2}-1\right) \equiv x^{n_{0}}(x-1)^{2} \equiv 0(c(x), P)$ we see that $r(x) \equiv 0(c(x), P)$. Hence, $r(x)$ is divisible by $c(x) \bmod P$, which implies $r_{k} \equiv 0(P)$ for all $k$, since the degree of $r(x) \bmod P$ is smaller than the degree of $c(x) \bmod P \quad(=\operatorname{deg}(c(x))$ since the leading coefficient is 1$)$. Observing that $x^{n_{0}}\left(x^{2 k}-1\right) \equiv 0(c(x), P)$, we see that this implies

$$
\begin{aligned}
x^{n_{0}} r(x)^{2} & \equiv x^{n_{0}} \sum r_{k}^{2} x^{2 k} \equiv \sum r_{k}^{2}\left(x^{n_{0}}\left(x^{2 k}-1\right)+x^{n_{0}}\right) \\
& \equiv x^{n_{0}} \sum r_{k}^{2} \equiv x^{n_{0}}\left(\sum r_{k}\right)^{2}\left(c(x), P^{3}\right)
\end{aligned}
$$


From $x^{n_{0}}\left(x^{2}-1\right) \equiv r(x) \bmod (c(x))$ we deduce

$$
x^{2 n_{0}}\left(x^{2}-1\right)^{2} \equiv r(x)^{2} \bmod (c(x)) .
$$

Hence,

$$
\begin{aligned}
x^{3 n_{0}}\left(x^{4}-1\right) & =2 x^{3 n_{0}}\left(x^{2}-1\right)+x^{3 n_{0}}\left(x^{2}-1\right)^{2} \equiv 2 x^{3 n_{0}}\left(x^{2}-1\right)+x^{n_{0}} r(x)^{2} \\
& \equiv 2 x^{3 n_{0}}\left(x^{2}-1\right)+x^{n_{0}}\left(\sum r_{k}\right)^{2}\left(c(x), P^{3}\right)
\end{aligned}
$$

and, by Lemma 1 ,

$$
u_{j+3 n_{0}+4}-u_{j+3 n_{0}} \equiv 2\left(u_{j+3 n_{0}+2}-u_{j+3 n_{0}}\right)+u_{j+n_{0}}\left(\sum r_{k}\right)^{2}\left(P^{3}\right) \text {. }
$$

From $x^{n_{0}}\left(x^{2}-1\right) \equiv 0(c(x), P)$ and

$$
x^{2 n_{0}}\left(x^{4}-1\right) \equiv 2 x^{2 n_{0}}\left(x^{2}-1\right)+x^{2 n_{0}}\left(x^{2}-1\right)^{2} \equiv 0\left(c(x), P^{2}\right),
$$

we see that $u_{j+2} \equiv u_{j}(P)$ and $u_{j+4} \equiv u_{j}\left(P^{2}\right)$ for $j \geqslant j_{0}=2 n_{0}$. Since $\left(u_{n}\right)$ is u.d. $\bmod P^{2}$, each of the four residues $\bmod P^{2}$ must appear once in a period, which implies $u_{j+2} \not \equiv u_{j}\left(P^{2}\right)$ for $j \geqslant j_{0}$, i.e., $u_{j+2}-u_{j}$ lies in the unique residue class mod $P^{2}$ that belongs to $P$ but not to $P^{2}$. We conclude that $u_{j+3}-u_{j+1} \equiv u_{j+2}-u_{j}$ $\left(P^{2}\right)$ for $j \geqslant j_{0}$. Since $u_{j+1}-u_{j} \equiv 1(P)$, we finally obtain

$\sum r_{k} \equiv \sum r_{k}\left(u_{j+1+k}-u_{j+k}\right) \equiv\left(u_{j+n_{0}+3}-u_{j+n_{0}+1}\right)-\left(u_{j+n_{0}+2}-u_{j+n_{0}}\right) \equiv 0\left(P^{2}\right)$ (taking into account that $x^{n_{0}}\left(x^{2}-1\right) \equiv \sum r_{k} x^{k} \bmod (c(x))$ implies $u_{j+n_{0}+2}-u_{j+n_{0}}$ $\left.\equiv \sum r_{k} u_{j+k}\right)$. Hence, $\left(\sum r_{k}\right)^{2} \equiv 0\left(P^{3}\right)$ and

$$
u_{j+3 n_{0}+4}-u_{j+3 n_{0}} \equiv 2\left(u_{j+3 n_{0}+2}-u_{j+3 n_{0}}\right)\left(P^{3}\right) \text {. }
$$

If $2 \equiv 0\left(P^{2}\right)$, this means that $\left(u_{n}\right)$ has period $4 \bmod P^{3}$; since 4 is not divisible by $N\left(P^{3}\right)=2^{3},\left(u_{n}\right)$ is not u.d. $\bmod P^{3}$. Now let us assume $2 \neq 0\left(P^{2}\right)$; then the above relation yields $u_{j+4}-u_{j} \neq 0\left(P^{3}\right)$ for sufficiently large $j$. Theorem 1 now gives $\left(I=P, a=2, h_{0}=1, l=2, k=1\right)$

$$
u_{j+2^{h+1}} \equiv u_{j}+2^{h-1}\left(u_{j+4}-u_{j}\right)\left(P^{h+2}\right) \text { for } h>0, j \geqslant 3 \cdot 2^{h-1} n_{0} .
$$

Hence,

$$
u_{j+2^{h+1}} \equiv u_{j}\left(P^{h+1}\right) \quad \text { and } \quad u_{j+2^{h+1}} \neq u_{j}\left(P^{h+2}\right) \quad \text { for } j \geqslant j_{0}(h), h>0 .
$$

By assumption, $\left(u_{n}\right)$ is u.d. $\bmod P^{2}$, i.e., every residue appears once in a period of length 4 . Since $u_{j+4} \neq u_{j}\left(P^{3}\right)$, this implies that every residue $\bmod P^{3}$ appears once in a period of length 8 . Inductively we conclude the analogous statement modulo $P^{h}$ for all $h$, i.e., $\left(u_{n}\right)$ is u.d. $\bmod P^{h}$ for all $h$.

THEOREM 2. Let I be a proper ideal of the Dedekind domain $R$ and let $\left(u_{n}\right)$ be a linear recurring sequence (of elements of $R$ ) with characteristic polynomial $c(x)$. Assume that for every prime divisor $P$ of $I, c(x)$ splits into linear factors modulo $P$ and that the factors incongruent to $x$ appear with multiplicity at most two. Then $\left(u_{n}\right)$ is u.d. $\bmod I$ if and only if the following conditions hold:

(1) If $P \mid I$ then $\left(u_{n}\right)$ is $u . d . \bmod P$; if $P^{2} \mid I$ and $p=2$ or $p=3$, then $\left(u_{n}\right)$ is u.d. $\bmod P^{2}$.

(2) If $P^{2} \mid I$ and $p \geqslant 5$, then $p \not \equiv 0\left(P^{2}\right)$; if $P^{3} \mid I$ and $p=2$ or $p=3$, then $p \not \equiv 0$ $\left(P^{2}\right)$.

(3) If $P_{i} \mid I(i=1,2)$ and $P_{1} \neq P_{2}$, then $N\left(P_{1}\right) \neq N\left(P_{2}\right)$. 
Proof. We assume first that $\left(u_{n}\right)$ is u.d. $\bmod I$. Then $\left(u_{n}\right)$ is u.d mod every divisor of $I$. This proves (1). For $P^{2} \mid I$ and $p \geqslant 5$ we deduce

$$
u_{j+p(p-1)} \equiv u_{j}+p\left(u_{j+p-1}-u_{j}\right)\left(P^{2}\right)
$$

from Lemma 3(c). Assume $p \equiv 0\left(P^{2}\right)$; then $u_{j+p(p-1)} \equiv u_{j}\left(P^{2}\right)$ (for sufficiently large $j$ ), which is impossible since the period must be divisible by $N\left(P^{2}\right)=p^{2}$. If $P^{3} \mid I$ and $p=2$, the preceding lemma implies $p \not \equiv 0\left(P^{2}\right)$. For the remaining case $p=3$ we use Lemma 3(b) to obtain

$$
u_{j+p^{2}(p-1)} \equiv u_{j}+p\left(u_{j+p(p-1)}-u_{j}\right)\left(P^{3}\right)
$$

so that

$$
u_{j+p^{2}(p-1)} \equiv u_{j}\left(P^{3}\right) \text { if } p \equiv 0\left(P^{2}\right) .
$$

Since $p^{2}(p-1)$ is not divisible by $N\left(P^{3}\right)=p^{3},\left(u_{n}\right)$ cannot be u.d. $\bmod P^{3}$; hence $P^{3}$ cannot divide $I$. This concludes the proof of (2).

Assume $P_{i} \mid I(i=1,2)$ and $P_{1} \neq P_{2}$; then $P_{1} P_{2}$ divides $I$. Hence, the period of $\left(u_{n}\right)$ modulo $P_{1} P_{2}$ must be divisible by $N\left(P_{1} P_{2}\right)$. By Lemma 3(a) we have $N\left(P_{i}\right)=p_{i}$ and $\left(u_{n}\right)$ has period $p_{i}\left(p_{i}-1\right) \bmod P_{i}$. From $N\left(P_{1}\right)=N\left(P_{2}\right)$ we obtain $p_{1}=p_{2}=$ $p$, so that $\left(u_{n}\right)$ has period $p(p-1) \bmod P_{1} P_{2}=P_{1} \cap P_{2}$. Since $p(p-1)$ is not divisible by $N\left(P_{1} P_{2}\right)=p^{2}$, we arrive at a contradiction. Hence, $N\left(P_{1}\right) \neq N\left(P_{2}\right)$.

Now we assume (1)-(3). If $P \mid I$ then, by $(1),\left(u_{n}\right)$ is u.d. $\bmod P$, so that Lemma 3(a) shows $N(P)=p$ and $u_{j+p^{h}(p-1)} \equiv u_{j}\left(P^{h}\right)$ for $h \geqslant 1, j \geqslant j_{0}(h)$. By (3), a rational prime $p$ belongs only to one prime ideal. Hence, every divisor of $I$ may be written in the form $\Pi P_{i}^{h_{i}} \cdot P^{k}, p_{1}<p_{2}<\cdots<p ; \Pi P_{i}^{h_{i}}$ may be the empty product. In order to prove that $\left(u_{n}\right)$ is u.d. $\bmod I$, we may therefore proceed inductively and show that $\left(u_{n}\right)$ is u.d. $\bmod \Pi P_{i}^{h_{i}} \cdot P^{k+1}$ provided $\left(u_{n}\right)$ is u.d. mod $\Pi P_{i}^{h_{i}} \cdot P^{k}, P^{k+1} \mid I$, and $p_{1}<p_{2}<\cdots<p$. The first step is given by the first part of (1). If $p=2$, the assertion follows from (1),(2), and Lemma 4. In the following we assume $p>2$. Define $l=\Pi p_{i}^{h_{i}}\left(p_{i}-1\right)$; then $\left(u_{n}\right)$ has period $l$ modulo $\cap P_{i}^{h_{i}}=$ $\Pi P_{i}^{h_{i}}$, so that $u_{j+n l}-u_{j} \equiv n\left(u_{j+l}-u_{j}\right)\left(\Pi P_{i}^{h_{i}}\right)$ for sufficiently large $j$. By Lemma 3 we have

$$
u_{j+n p^{k}(p-1)}-u_{j} \equiv n\left(u_{j+p^{k}(p-1)}-u_{j}\right)\left(P^{k+1}\right) \quad \text { for } k \geqslant 0, j \geqslant j_{0}(k) .
$$

Hence,

$$
u_{j+n l p^{k}(p-1)}-u_{j} \equiv n\left(u_{j+l p^{k}(p-1)}-u_{j}\right)\left(\Pi P_{i}^{h_{i}} \cdot P^{k+1}\right) .
$$

If we can prove $u_{j+l p^{k}(p-1)}-u_{j} \neq \equiv\left(P^{k+1}\right)$, then the last congruence means that $u_{j+n l p^{k}(p-1)}(n=0, \ldots, p-1)$ runs through the $p$ residues $\bmod \Pi P_{i}^{h_{i}} \cdot P^{k+1}$ belonging to the residue $u_{j} \bmod \Pi P_{i}^{h_{i}} \cdot P^{k}$. Since $\left(u_{n}\right)$ has period $l p^{k}(p-1) \bmod \Pi P_{i}^{h_{i}} \cdot P^{k}$, this implies that $\left(u_{n}\right)$ is u.d. $\bmod \Pi P_{i}^{h_{i}} \cdot P^{k+1}$ provided $\left(u_{n}\right)$ is u.d. $\bmod \Pi P_{i}^{h_{i}} \cdot P^{k}$. From $u_{j+l p^{k}(p-1)}-u_{j} \equiv l\left(u_{j+p^{k}(p-1)}-u_{j}\right)\left(P^{k+1}\right)$ and $(l, p)=1$ (since $p_{i}<p$ for all $i$ ), we see that it remains to prove $u_{j+p^{k}(p-1)}-u_{j} \neq \equiv$ $\left(P^{k+1}\right)$. If $k=0$, this follows from Lemma 3(a). If $k \geqslant 1$ and $p \geqslant 5$ we have, by Lemma 3,

$$
u_{j+p^{k}(p-1)}-u_{j} \equiv p^{k-1}\left(u_{j+p(p-1)}-u_{j}\right) \equiv p^{k}\left(u_{j+p-1}-u_{j}\right)\left(P^{k+1}\right) .
$$


Since, by (2), $p \not \equiv 0\left(P^{2}\right)$ and $u_{j+p-1}-u_{j} \not \equiv 0(P)$, this proves the assertion in this case. Now suppose $p=3$. By $(1),\left(u_{n}\right)$ is u.d. $\bmod P^{2}$. If $u_{j+p(p-1)}-u_{j} \equiv 0\left(P^{2}\right)$ for some $j \geqslant j_{0}$, then $u_{j+n p(p-1)}-u_{j} \equiv n\left(u_{j+p(p-1)}-u_{j}\right)\left(P^{2}\right)$ implies that the residue $u_{j}$ appears $p$ times (for $n=0, \ldots, p-1$ ) in a period of length $p^{2}(p-1)$. Since there are $p^{2}$ residues $\bmod P^{2}$, each of them must appear $(p-1)$ times. Hence, $u_{j+p(p-1)}-u_{j} \not \equiv 0\left(P^{2}\right)$ for all sufficiently large $j$. Consequently, the assertion is equivalent to $p^{k-1} \not \equiv 0\left(P^{k}\right)$, i.e., $p \not \equiv 0\left(P^{2}\right)$ if $k>1$. To conclude the proof we remark that $k>1$ and $P^{k+1} \mid I$ imply $P^{3} \mid I$; hence, $p \not \equiv 0\left(P^{2}\right)$ follows from (2).

REMARK. (1) Let $\left(u_{n}\right)$ be a linear recurring sequence with arbitrary characteristic polynomial $c(x)$, and let $P$ be a prime ideal with finite norm. Assume that $c(x)$ has no multiple factors mod $P$ except possibly the factor $x$, whose multiplicity we denote by $n_{0}$. If $d$ is the degree of the splitting field of $c(x)$ over $R / P, c(x)$ divides $x^{n_{0}}\left(x^{N(P)^{d}-1}-1\right) \bmod P$, i.e., $x^{n_{0}}\left(x^{N(P)^{d}-1}-1\right) \equiv 0(c(x), P)$. Then, by Lemma 1 , $\left(u_{n}\right)$ has period $N(P)^{d}-1 \bmod P$. Since this number is not divisible by $N(P),\left(u_{n}\right)$ cannot be u.d. $\bmod P$. Hence, in order that $\left(u_{n}\right)$ be u.d. $\bmod P, c(x)$ must have a nontrivial multiple factor $\bmod P$.

(2) Conditions (2) and (3) of the theorem are satisfied trivially if $R$ is the ring of rational integers.

4. The case $\operatorname{deg}(c(x))=2$. Apart from a substantial simplification of the proof of Lemma 4, restriction to second-order linear recurring sequences would only have entailed minor simplifications (mainly due to the fact that we could assume $n_{0}=0$ ) in the preceding investigations. The restriction is essential for the following complete classification, however.

Theorem 3. Let I be a proper ideal of the Dedekind domain $R$ and let $\left(u_{n}\right)$ be a linear recurring sequence (of elements of $R$ ) with characteristic polynomial $c(x)=$ $x^{2}-c_{1} x-c_{0}$. Then $\left(u_{n}\right)$ is u.d. $\bmod I$ if and only if the following conditions hold:

(1) If $P \mid I$, then $N(P)=p, c_{1}^{2}+4 c_{0} \equiv 0(P), c_{0} \not \equiv 0(P) ; 2 u_{1} \not \equiv c_{1} u_{0}(P)$ for $p>2, u_{1} \neq u_{0}(P)$ for $p=2$. If $P^{2} \mid I$ and $p=2$, then $c_{1} \neq \equiv 0\left(P^{2}\right), c_{0} \not \equiv 1\left(P^{2}\right)$; if $P^{2} \mid I$ and $p=3$ then $c_{0}+c_{1}^{2} \neq 0\left(P^{2}\right)$.

(2) If $P^{2} \mid I$ and $p \geqslant 5$, then $p \neq \equiv\left(P^{2}\right)$; if $P^{3} \mid I$ and $p=2$ or $p=3$, then $p \neq \equiv$ $\left(P^{2}\right)$.

(3) If $P_{i} \mid I(i=1,2)$ and $P_{1} \neq P_{2}$, then $N\left(P_{1}\right) \neq N\left(P_{2}\right)$.

Proof. By part (1) of the last remark we only have to show that (1) is equivalent to condition (1) of Theorem 2 . Let $P$ be a prime ideal. If $\left(u_{n}\right)$ is u.d. mod $P$ then $N(P)=p$ by Lemma 3(a). The conditions $c_{0} \equiv \equiv(P)$ and $c_{1}^{2}+4 c_{0} \equiv 0(P)$ again follow from the above-cited remark since they are equivalent to $c(x) \equiv(x-a)^{2}$ $(P)$ for some $a \neq 0(P)$. From $c(x) \equiv(x-a)^{2}$ we conclude

$$
a u_{n} \equiv\left(\left(u_{1}-a u_{0}\right) n+a u_{0}\right) a^{n}(P) .
$$

Since $a^{p-1}=a^{N(P)-1} \equiv 1(P)$, we obtain

$$
a\left(u_{j+n(p-1)}-u_{j}\right) \equiv n\left(u_{1}-a u_{0}\right)(p-1) a^{j} \equiv-n\left(u_{1}-a u_{0}\right) a^{j}(P) .
$$


If $u_{1}-a u_{0} \equiv 0(P)$, this means that $\left(u_{n}\right)$ has period $p-1 \bmod P$; hence $\left(u_{n}\right)$ is not u.d. $\bmod P$. If $u_{1}-a u_{0} \neq 0(P)$, then the subsequences $\left(u_{j+n(p-1)}\right)(j=$ $0, \ldots, p-2)$ are u.d. $\bmod P$, being nontrivial arithmetic sequences $\bmod P$. Since $\left(u_{n}\right)$ is the union of these subsequences, we finally obtain that $\left(u_{n}\right)$ is u.d. $\bmod P$ if and only if $u_{1}-a u_{0} \equiv 0(P)$. For $p=2$ this means $u_{1}-u_{0} \not \equiv 0(P)$; for $p>2$ the condition is equivalent to $2 u_{1} \not \equiv c_{1} u_{0}(P)$ since $2 \not \equiv 0(P)$ and $2 a \equiv c_{1}(P)$.

Let $\left(u_{n}\right)$ be u.d. $\bmod P$. Assume $p=2$ first. We have to show that $\left(u_{n}\right)$ is u.d. $\bmod P^{2}$ if and only if $c_{0} \not \equiv 1\left(P^{2}\right)$ and $c_{1} \not \equiv 0\left(P^{2}\right)$. Since $\left(u_{n}\right)$ is u.d. $\bmod P$, we have $c_{0} \equiv 1(P), c_{1} \equiv 0(P)$, and $u_{j+1} \equiv u_{j}+1(P)$ for $j \geqslant 0$. By Lemma 3(a) and the following remark, $u_{j+4} \equiv u_{j}\left(P^{2}\right)$ for $j \geqslant 0$. Hence, $\left(u_{n}\right)$ is u.d. $\bmod P^{2}$ if and only if $u_{0} \not \equiv u_{2}\left(P^{2}\right)$ and $u_{1} \neq u_{3}\left(P^{2}\right)$. The second condition may be replaced by $u_{3}-u_{1} \equiv u_{2}-u_{0}\left(P^{2}\right)$. Since

$$
\begin{aligned}
\left(u_{3}-u_{1}\right)-\left(u_{2}-u_{0}\right) & =\left(c_{1} u_{2}+\left(c_{0}-1\right) u_{1}\right)-\left(c_{1} u_{1}+\left(c_{0}-1\right) u_{0}\right) \\
& \equiv c_{1}\left(u_{2}-u_{1}\right)+\left(c_{0}-1\right)\left(u_{1}-u_{0}\right) \equiv c_{1}+\left(c_{0}-1\right)\left(P^{2}\right)
\end{aligned}
$$

and

$$
\begin{aligned}
u_{2}-u_{0} & =c_{1} u_{1}+\left(c_{0}-1\right) u_{0} \equiv c_{1}\left(u_{0}+1\right)+\left(c_{0}-1\right) u_{0} \\
& \equiv\left(c_{1}+c_{0}-1\right) u_{0}+c_{1}\left(P^{2}\right)
\end{aligned}
$$

we obtain the conditions $c_{1}+\left(c_{0}-1\right) \equiv 0\left(P^{2}\right)$ and $c_{1} \neq \equiv\left(P^{2}\right)$, which are equivalent to $c_{0}-1 \neq \equiv\left(P^{2}\right)$ and $c_{1} \not \equiv 0\left(P^{2}\right)$.

Now assume $p=3$. We prove that $\left(u_{n}\right)$ is u.d. $\bmod P^{2}$ if and only if $c_{0}+c_{1}^{2} \not \equiv 0$ $\left(P^{2}\right)$. By Lemma 3 we have $u_{j+6} \equiv u_{j}(P)$ and $u_{j+6 n} \equiv u_{j}+n\left(u_{j+6}-u_{j}\right)\left(P^{2}\right)$ for $j \geqslant 0$. If, for some $j, u_{j+6}-u_{j} \equiv 0\left(P^{2}\right)$, then $u_{j}$ appears three times (for $n=0,1,2$ ) in a period of length 18; hence, $\left(u_{n}\right)$ is not u.d. $\bmod P^{2}$ in this case. If $u_{j+6}-u_{j} \neq 0$ $\left(P^{2}\right)$, then $u_{j+6 n}(n=0,1,2)$ runs through the three residues $\bmod P^{2}$ belonging to the residue class of $u_{j} \bmod P$. Since $\left(u_{n}\right)$ is u.d. $\bmod P$ and $u_{j+6} \equiv u_{j}(P)$, this implies that $\left(u_{n}\right)$ is u.d. $\bmod P^{2}$ provided $u_{j+6} \not \equiv u_{j}\left(P^{2}\right)$ for all $j$. As in the first part of the proof we have $c(x) \equiv(x-a)^{2}(P), a \equiv 0(P)$; hence $c_{0} \equiv-a^{2}(P)$, $c_{1} \equiv 2 a(P)$. From $c(x)=c(a)+\left(2 a-c_{1}\right)(x-a)+(x-a)^{2}$ we conclude that $c(a)(x-a)+(x-a)^{3} \equiv 0\left(c(x), P^{2}\right)$, since $\left(2 a-c_{1}\right) \equiv 0(P)$, and $(x-a)^{2} \equiv 0$ $(c(x), P)$. Observing that

$$
x^{3}-a^{3}=(x-a)^{3}+3 a x(x-a) \text { and } x(x-a) \equiv a(x-a)(c(x), P),
$$

we obtain

$$
x^{3}-a^{3} \equiv-c(a)(x-a)+3 a^{2}(x-a) \equiv\left(3 a^{2}-c(a)\right)(x-a)\left(c(x), P^{2}\right) .
$$

Since $x^{3}-a^{3} \equiv(x-a)^{3} \equiv 0 \quad(c(x), P)$ and $x^{3}+a^{3}=x^{3}-a^{3}+2 a^{3} \equiv 2 a^{3}$ $(c(x), P)$, this yields

$$
x^{6}-a^{6}=\left(x^{3}-a^{3}\right)\left(x^{3}+a^{3}\right) \equiv 2 a^{3}\left(3 a^{2}-c(a)\right)(x-a)\left(c(x), P^{2}\right) .
$$

From $a^{2}=a^{N(P)-1} \equiv 1(P)$ we obtain

$$
a^{6}=1+3\left(a^{2}-1\right)+3\left(a^{2}-1\right)^{2}+\left(a^{2}-1\right)^{3} \equiv 1\left(P^{2}\right) .
$$


Hence (by Lemma 1),

$$
u_{j+6}-u_{j} \equiv 2 a^{3}\left(3 a^{2}-c(a)\right)\left(u_{j+1}-a u_{j}\right)\left(P^{2}\right) .
$$

Since $u_{j+1}-a u_{j} \equiv a\left(u_{j}-a u_{j-1}\right) \equiv \cdots \equiv a^{j}\left(u_{1}-a u_{0}\right)(P)$ and $u_{1}-a u_{0} \equiv 0$ $(P), u_{j+6}-u_{j} \neq \equiv 0\left(P^{2}\right)$ is seen to be equivalent to $3 a^{2}-c(a) \neq \equiv 0\left(P^{2}\right)$. Taking $a=2 c_{1}$ ( $a$ was only subject to the condition $\left.c_{1} \equiv 2 a(P)\right)$, we finally conclude that $\left(u_{n}\right)$ is u.d. $\bmod P^{2}$ if and only if

$$
c_{1}^{2}+c_{0} \equiv 3 \cdot 4 c_{1}^{2}-\left(4 c_{1}^{2}-2 c_{1}^{2}-c_{0}\right) \not \equiv 0\left(P^{2}\right) .
$$

\section{REFERENCES}

1. R. T. Bumby, A distribution property for linear recurrence of the second order, Proc. Amer. Math. Soc. 50 (1975), 101-106.

2. P. Bundschuh and J.-S. Shiue, Solution of a problem on the uniform distribution of integers, Atti Accad. Naz. Lincei Rend. Cl. Sci. Fis. Mat. Natur. 55 (1973), 172-177.

3. M. J. Knight and W. A. Webb, Uniform distribution of third order linear recurrence sequences, Acta Arith. 36 (1980), 7-20.

4. L. Kuipers and J.-S. Shiue, $A$ distribution property of the sequence of Fibonacci numbers, Fibonacci Quart. 10 (1972), 375-376, 392.

5. __ A distribution property of the sequence of Lucas numbers, Elem. Math. 27 (1972), 10-11.

6. $\ldots$ A distribution property of a linear recurrence of the second order, Atti Accad. Naz. Lincei Rend. Cl. Sci. Fis. Mat. Natur. 52 (1972), 6-10.

7. W. Narkiewicz, Uniform distribution of sequences of integers in residue classes, Lecture Notes in Math., vol. 1087, Springer-Verlag, Berlin and New York, 1984.

8. M. B. Nathanson, Linear recurrences and uniform distribution, Proc. Amer. Math. Soc. 48 (1975), 289-291.

9. H. Niederreiter, Distribution of Fibonacci numbers mod 5 ${ }^{k}$, Fibonacci Quart. 10 (1972), 373-374.

10. H. Niederreiter and J.-S. Shiue, Equidistribution of linear recurring sequences in finite fields, Indag. Math. 39 (1977), 397-405.

11. , Equidistribution of linear recurring sequences in finite fields, II, Acta Arith. 38 (1980), 197-207.

12. P. Ribenboim, Algebraic numbers, Wiley, New York, 1972.

13. R. F. Tichy and G. Turnwald, Uniform distribution of recurrences in Dedekind domains, Acta Arith. 46 (1985), 81-89.

14. G. Turnwald, Gleichverteilung linearer Rekursionen, Dissertation, Techn. Univ. Wien, 1984.

15. Gleichverteilung von linearen rekursiven Folgen, Sitzungsber. Österr. Akad. Wiss. Math.Naturwiss. Kl. 193 (1984), 201-245.

16. W. A. Webb and C. T. Long, Distribution modulo $p^{h}$ of the general linear second order recurrence, Atti Accad. Naz. Lincei Rend. Cl. Sci. Fis. Mat. Natur. 58 (1975), 92-100.

Institut fÜ ANALysis, TeChNische Mathematik UND Versicherungsmathematik, Technische UNIVERSitÄt Wien, Wiedner Hauptstr, 8-10, A - 1040 VienNa, Austria 